\title{
Pénétrer la boîte noire du traducteur
}

\author{
Antin Fougner Rydning
}

Université d'Oslo

A combination of the two process-oriented approaches to studying professional translation online: (i) verbal reporting, better known as TAP (thinkaloud protocols), and (ii) Translog, a program used for logging keyboard activity, yields interesting data about what goes on in the translator's mind during a translation task. Assuming that our interpretation of metaphorical expressions depend upon conceptual metaphors, examples selected from the online data of two experienced professional Norwegian translators are used to show how the translation of metaphorical concepts are handled. Possible ways of using the above-mentioned online data qualitatively to test the hypothesis according to which translation is more than an interlinguistic comparison, requiring the mobilisation of cognitive complements in the reformulation phase, are also suggested.

\section{Introduction}

La présente étude qui s'inscrit dans le cadre d'une recherche en cours sur l'expertise en traduction, financé par le Conseil norvégien de recherche scientifique 2001-2004, vise à décrire l'activité cognitive liée à la traduction d'expressions métaphoriques à partir de quelques exemples extraits de données traductionelles in vivo, c'est-à-dire prises sur le vif pendant que le traducteur accomplit sa tâche de traduction. Prenant appui sur le modèle de la métaphore conceptuelle, tel qu'il a été développé e.a. par Lakoff \& Turner (1989), je propose de tester l'hypothèse de Lederer (1994) sur le rôle des compléments notionnels pour la reformulation réussie d'une idée: "L'équivalence cognitive découle de la jonction du sémantisme du texte et des compléments notionnels apportés par le traducteur." (52) Par compléments notionnels, Lederer entend les éléments pertinents du bagage cognitif, c'est-à-dire l'intégralité du savoir notionnel et émotionnel de l'individu (211), et du contexte cognitif, c'est-à-dire le savoir accumulé et conservé tout au long de la lecture du texte (42). Pour ce faire je me fonde sur les données processuelles in vivo de mon corpus, celles-ci permettant particulièrement bien de déceler les activités cachées dans la traduction finale.

\section{Le mécanisme de la métaphorisation conceptuelle}

Rendons brièvement compte de la conception cognitiviste de la métaphore conceptuelle, avant d'illustrer comment les méthodes processuelles in vivo TAP et Translog (voir 3. ci-dessous) permettent de pénétrer la boîte noire du traducteur. 


\subsection{La métaphore conceptuelle}

Selon la conception cognitiviste de Lakoff \& Johnson (1980, 1984), Lakoff \& Turner (1989), Gibbs (1994), notre système conceptuel ordinaire, c.à.d. celui qui nous permet de penser et d'agir, est de nature fondamentalement métaphorique. La métaphore n'a rien d'unique, d'original ou d'ornemental. La métaphore n'est pas une déviation par rapport à ce qui est normal dans le langage. En fait, il n'y a rien de plus normal que la métaphore - et les expressions métaphoriques fourmillent dans le langage. Elles sont plutôt la règle que l'exception. Ne pouvant - en raison de contraintes d'espace - entrer dans le détail de la métaphore conceptuelle, contentons-nous de citer un passage représentatif de celle-ci chez Lakoff \& Turner:

Basic conceptual metaphors are part of the common conceptual apparatus shared by members of a culture. They are systematic in that there is a fixed correspondence between the structure of the domain to be understood (e.g., death) and the structure of the domain in terms of which we are understanding it (e.g., departure). We usually understand them in terms of common experiences. They are largely unconscious, though attention may be drawn to them. Their operation in cognition is mostly automatic. And they are widely conventionalized in language, that is, there are a great number of words and idiomatic expressions in our language whose interpretations depend upon those conceptual metaphors. (51)

Comme la pensée métaphorique est réfléchie dans le langage, il est possible de distinguer entre (i) le concept métaphorique qui est une image abstraite et (ii) l'expression métaphorique qui est la retombée linguistique de notre façon de concevoir l'image abstraite.

\subsection{Illustration de la métaphore conceptuelle}

Illustrons comment la saisie du concept métaphorique du consensus peut s'expliquer en termes de projection domaniale à partir de l'énoncé inédit (1) ci-dessous:

(1) les bonnes manières sont à nouveau plébiscitées par les Français.

Cet énoncé est tiré du texte ci-après (2) extrait de l'Express:

(2) Le retour des bonnes manières

On les croyait désuètes, balayées par l'ouragan post-soixantehuitard, promises à une mort imminente par la recrudescence des incivilités. On les imaginait figées, tout juste bonnes à illustrer les manuels d'éducation pour jeunes filles de bonne famille. Erreur. Liftées, épurées, les bonnes manières sont à nouveau plébiscitées par les Français. Les bonnes manières sont un signe d'intégration. Mais elles restent aussi, plus sournoisement, un mode de tri social. Raison de plus pour maîtriser les codes. Les entreprises exigent 
aujourd'hui de leurs employés qu'ils sachent communiquer. Le "BSAM" (bonjour-sourire-au revoir-merci) est enseigné partout. Conseillère en image personnelle, Hélène Choumiloff veille au grain. Ses "élèves" sont des cadres de sexe masculin de plus de 40 ans. Leur objectif: éviter les gaffes au cours des dîners d'affaires. "Beaucoup de mes clients se sont faits à la force du poignet. C'est l'ascension sociale qui crée la gêne", constate la conseillère. Pendant les exercices, dans la rue ou au restaurant, elle traque les failles. L'apprentissage peut durer trois mois. Les élèves sont très motivés. S'il est désormais bien vu de laisser au placard cravate et costume trois pièces le vendredi, nul ne tolère, désormais, les infractions au code de bonne conduite. (Laurence Albert.) Extrait de l'Express, Société, du 4-11 janvier 2000.

Grâce à notre connaissance générale du recours au plébiscite, nous sommes à même d'actualiser une projection entre le domaine source du code civil et le domaine cible $d u$ code de conduite, projection qui nous permet de procéder à une série de déductions, et d'inférer p. ex. qu'un vote majoritaire entraîne l'adoption d'une loi. Lorsque ce domaine est utilisé pour une projection métaphorique, le réseau d'inférences du domaine source est automatiquement projeté sur le domaine cible, et les aspects pertinents de la projection sont actualisés par le contexte. Cette projection nous permet de retenir que, puisque la plupart des Français sont en faveur des bonnes manières, ces dernières domineront dorénavant la scène dans les rapports sociaux. Soit le schéma suivant illustrant la représentation conceptuelle de la métaphore UN CODE DE CONDUITE EST UN CODE CIVIL:

(3)

\begin{tabular}{|l|l|}
\hline Domaine source & Domaine cible \\
\hline CODE CIVIL & CODE DE BONNE CONDUITE \\
\hline Lois & Règles de conduite \\
\hline Corps d'électeurs & Les Français \\
\hline Plébiscite & Consultation \\
\hline Dire oui & Être en faveur de \\
\hline Adopter & Faire sien \\
\hline
\end{tabular}

\section{Exposition des deux méthodes de collection de données in vivo: TAP et Translog}

\subsection{La méthode TAP (think-aloud-protocols)}

La méthode de collection de données verbales et comportementales prises sur le vif pendant l'opération traduisante, dite la méthode TAP, est importée 
de la psychologie cognitive et appliquée à la traduction non professionnelle au milieu des années 80 . Ce n'est que vers la fin des années 80 , que la méthode TAP commence à être appliquée à la traduction professionnelle (Tirkkonen-Condit 1987; Jääskelaïnen 1987; Séguinot 1989). Elle consiste à demander au traducteur de dire tout haut ce qu'il pense tout bas pendant qu'il traduit. Ses commentaires verbaux ainsi que son comportement non verbal sont enregistrés sur vidéo. Ces données spontanées sont par la suite transcrites en protocoles de verbalisation, appelés TAP dans la littérature traductologique anglophone. Eu égard aux grandes variations individuelles en ce qui concerne la verbalisation portant sur une activité écrite complexe et exigeante - certains sujets verbalisant beaucoup, d'autres peu - les données TAP doivent être traitées avec discernement. (Pour une bibliographie annotée des études TAP en traduction ainsi qu'une réponse aux critiques qui ont été adressées aux recherches processuelles fondées sur l'approche TAP, voir Jääskeläinen 2002). La pauvreté ou l'absence de commentaires verbaux est parfois très utilement compensée par le comportement non verbal du sujet (gestes, expressions faciales), qui peut être tout aussi révélateur d'un effort cognitif que les commentaires verbaux (cf. Rydning 2001, Bernardini 2000). Dans certains cas la gesticulation inconsciente du sujet traduisant peut précéder une solution. Voir à ce sujet l'exemple (5.8) ci-dessous.

\subsection{Illustration de l'emploi de la méthode TAP}

Un exemple puisé dans mon corpus servira à illustrer comment les commentaires TAP parviennent à jeter la lumière sur le problème de la reformulation dans la langue d'arrivée, phénomène resté dans l'ombre en traductologie. Rapportons (dans ma traduction française) les propos de Christine, expert-traducteur chevronné avec plus de 10 ans d'expérience active de la traduction professionnelle suite à une formation à la traduction, au sujet de la traduction de l'énoncé (1). Les gestes qui ponctuent ses commentaires TAP sont indiqués entre parenthèses.

(4) Les bonnes manières, vêtues plus simplement, font leur rentrée et sont bien accueillies. Non, je vais voir si mon dictionnaire peut me mettre sur la voie. Le sens propre de 'plébisciter' ne convient pas ici, voyons...(Consulte son dictionnaire) Non, ça n'a aucun rapport. (Ferme son dictionnaire) Sont accueillies... (Ouvre les bras) ... Et reçues à bras ouverts. Etre reçu à bras ouverts. Et sont reçues à bras ouverts, être reçu à bras ouverts, sont reçues... J'opte pour cette solution, qui est une espèce de personnification: des vêtements seyants. Sont reçues à bras ouverts par les Français.

Les commentaires TAP de Christine nous permettent de décomposer sa démarche cognitive liée à la recherche d'une solution de reformulation en 10 unités: 
(5) Composantes de la démarche cognitive de Christine

5.1 La formulation spontanée en norvégien de l'idée comprise

5.2 Le rejet de la solution initiale

5.3 L'identification du terme problématique 'plébiscitées'

5.4 Le rejet du transcodage de ce terme en norvégien

5.5 Le recours au dictionnaire

5.6 L'abandon du dictionnaire

5.7 La formulation d'une solution provisoire: hilse velkommen 'souhaiter la bienvenue'

5.8 Le geste qui consiste à ouvrir les bras

5.9 La formulation d'une seconde solution: tatt imot med åpne armer 'sont reçues à bras ouverts'

5.10 L'analyse justificative de la solution retenue

Il est intéressant de constater que l'analyse justificative dans (5.10) s'effectue au niveau conceptuel. Christine est parfaitement consciente d'avoir eu recours au procédé métaphorique de la personnification, selon lequel un concept abstrait est compris comme s'il était une personne. Les bonnes manières peuvent donc être représentées comme si elles étaient des personnes que les Français reçoivent à bras ouverts: LES BONNES MANIËRES SONT DES PERSONNES.

La traduction de Christine est réussie, car elle parvient à restituer très clairement l'idée de l'original. Les moyens linguistiques autres, certes, que ceux utilisés dans l'original, sont la retombée de deux façons différentes de concevoir l'idée abstraite du consensus. La différence conceptuelle entre la métaphore du texte de départ et la métaphore du texte d'arrivée montre qu'une seule et même personne est parfaitement capable de se représenter une scène à partir d'images conceptuelles différentes. C'est ce qu'exprime aussi Langacker: "People have the capacity to construe a scene by means of alternative images." (1991:35) L'avantage indéniable de la traduction réussie - celle qui garantit l'invariance du sens - est qu'elle permet d'observer, beaucoup plus clairement qu'au sein de la communication unilingue, cette capacité humaine de s'imaginer une scène au moyen de représentations conceptuelles plurielles.

Ajoutons une remarque sur le comportement non verbal de Christine. A la recherche d'une formulation susceptible de restituer l'idée déverbalisée du consensus, Christine ouvre les bras, avant d'accompagner ce geste de l'expression linguistique correspondante: 'sont reçues à bras ouverts'. La composante (5.8) ci-dessus relevée dans la démarche cognitive de Christine révèle que la reformulation de l'idée s'effectue en fonction d'une conceptualisation métaphorique qui précède l'expression linguistique. En attendant de soumettre cette hypothèse à une vérification empirique fondée sur l'analyse d'un corpus plus ample, retenons que le comportement non verbal du sujet traduisant (gesticulation, expressions faciales, réflexes) peut signaler un effort cognitif aussi révélateur que ses commentaires verbaux. 


\subsection{La méthode Translog}

Le programme informatique Translog, développé par Jakobsen \& Schou (1999), enregistre toutes les activités d'écriture et toutes les pauses du sujet traduisant lors de l'exécution de sa traduction sur son ordinateur. L'avantage de Translog est qu'il permet de suivre de façon à la fois objective et précise le mode naturel de travail du traducteur: ses solutions, ses corrections, les pauses précédant une activité d'écriture ainsi que sa façon de se mouvoir dans le texte. Les données informatisées sont représentées graphiquement ainsi qu'en version cinématique. Cette dernière permet de visualiser, de façon linéaire, les données enregistrées en temps réel, réduit ou accéléré. Dans la version graphique, générée automatiquement, chaque touche du clavier autre que celles qui correspondent aux lettres de l'alphabet est représentée au moyen d'un symbole. La version graphique imprimée sert de base, avec la version cinématique, à l'analyse interprétative du chercheur. Ce dernier peut à son gré déterminer la longueur des pauses en fonction du type d'analyse qu'il souhaite conduire (voir les différentes possibilités à cet égard: Jakobsen 2000:164-167). Si les pauses sont les indicateurs les plus évidents des efforts de résolution de problèmes liés à la production d'énoncés: "In cognitive psychology, pause analysis is an important factor in realtime research on language production as pauses are believed to reflect cognitive processes" (Jensen 2000:101), il s'ensuit qu'elles conviennent tout aussi bien pour détecter les problèmes de reformulation ressentis par le traducteur.

(6) Légende des symboles les plus courants

\begin{tabular}{|c|c|}
\hline$\star$ & unité de pause \\
\hline$[\star: n . n]$ & unité de pause étendue \\
\hline$\Leftrightarrow$ & touche interligne \\
\hline - & barre espace \\
\hline$\Delta \square$ & touche d'effacement à gauche ou à droite \\
\hline [Ctrl] & touche contrôle \\
\hline$\leftarrow \downarrow \uparrow \rightarrow$ & touches flèches \\
\hline [Shft] & touche majuscule \\
\hline
\end{tabular}

Dans l'extrait ci-dessous (7) du fichier Translog d'Anne - expert-traducteur ayant une formation et une pratique professionnelle de la traduction équivalente à celle de Christine - l'astérique correspond à une pause de 5 secondes. L'extrait représente sa traduction du titre du texte (2): Le retour aux bonnes manières:

(7) Données Translog

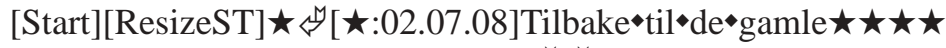

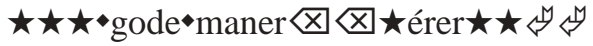


(8) Version transcodée des données Translog Tilbake til de gamle gode manérer 'Retour aux bonnes vieilles manières'

Aussitôt après l'apparition du texte à traduire sur son écran, Anne marque une pause initiale de 5 secondes $(\star)$, avant d'aller à la ligne $\left(\xi^{\mathfrak{y}}\right)$. La phase d'orientation initiale chez Anne dure 2 minutes, 7 secondes et 8 millisecondes [ $\star: 02.07 .08]$. Après avoir inscrit les 4 premiers mots sur son écran (Tilbake til de gamle 'Retour aux vieilles') elle effectue une pause de 35 secondes $(\star \star \star \star \star \star \star \star)$, puis écrit gode maner 'bonnes maniè', efface les deux dernières lettres $(\otimes \otimes)$, marque une nouvelle pause de 5 secondes ( $\star$ ) et remplace les deux lettres effacées par (érer). La dernière pause de 10 secondes $(\star \star)$ ) est suivie d'un double interligne $\left(\xi^{\sharp} \xi\right)$.

La longue pause initiale est consacrée à la lecture et à la compréhension du texte original. La pause initiale peut parfois aussi correspondre à la planification de la reformulation (voir l'exemple (11) ci-dessous), mais selon la définition adoptée par Jakobsen, aucune activité d'écriture n'a lieu dans la phase d'orientation initiale: "Some cognitive preparation of target production may be involved, but in our definition there can be no typing of target text in this phase" (2003 à venir). La phase de reformulation proprement dite commence donc dès lors qu'Anne se met à écrire. J'interprète la pause de 35 secondes qui suit l'inscription des 4 premiers mots sur son écran, comme un effort de réflexion soutenue pour répondre à la question de savoir si l'ajout du terme gamle 'vieilles' par rapport au texte original français se défend (Rydning 2003 à venir). Visiblement convaincue que sa solution est motivée, Anne termine la traduction du titre, tout en effectuant une correction en vue de se conformer à l'orthographe francisée de 'manières' en norvégien: manérer. La dernière pause de 10 secondes signale qu'elle se relit et qu'elle évalue sa traduction. Apparemment satisfaite, elle appuie sur la touche interligne deux fois, signe qu' elle est prête à passer à la traduction du texte proprement dit.

\subsection{L'avantage pratique de combiner l'approche Translog à l'approche TAP}

Les deux méthodes de collection des données processuelles in vivo se combinent parfaitement. Bien que le logiciel Translog ne soit pas équipé d'une fonction de synchronisation électronique permettant d'aligner les données Translog aux données TAP, la synchronisation manuelle est possible. Elle permet au chercheur de suivre les activités d'écriture en parallèle avec les commentaires verbaux, ce qui facilite l'interprétation qualitative des données. Prenons l'exemple suivant extrait du fichier Translog de Christine (9) suivi d'un transcodage français (10) et de ses commentaires TAP (11) en guise d'illustration: 
(9) Données Translog

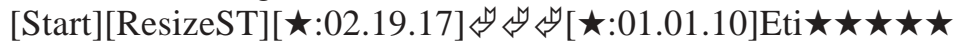

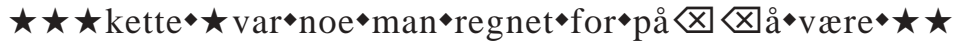
avleggs, $\bullet$ feiet $\bullet$ bort $\bullet$ ikjølvannet $\bullet$ av $\star \star \star \star 68 \bullet$

(10) Transcodage des données Translog

Le bon usage était considéré comme quelque chose de désuet, balayé dans le sillage de 68

Nous constatons que Christine fait l'impasse sur la traduction du titre. Ses commentaires TAP révèlent la raison de cette omission:

(11) Commentaires TAP

'Le retour des bonnes manières.' Je peux donc choisir de garder bonnes manières ou d'employer étiquette. (Regarde par la fenêtre) Je crois que je vais laisser le titre de côté pour l'instant, et plutôt attaquer le texte proprement dit.

Dans des cas moins clairs, où le chercheur hésite sur l'interprétation à donner à une pause Translog, les données TAP peuvent lui apporter le complément d'information requis et, inversement, les données Translog peuvent l'éclairer lorsque les commentaires TAP du sujet traduisant sont parcimonieux, voire même absents. Mais en plus de la simple description du mode de traduction du sujet traduisant, ce type de données processuelles in vivo présente l'avantage de pouvoir servir de base à la vérification empirique d'hypothèses.

\subsection{L'avantage théorique de disposer de données processuelles in vivo pour mettre en évidence l'activité mentale du traducteur}

Illustrons à partir des deux exemples (12) et (15) ci-dessous une démarche qui pourrait servir à tester l'assertion de l'Ecole de Paris selon laquelle la comparaison interlinguistique ne constitue pas une méthode de traduction: "La comparaison interlinguistique n'intervient pas dans la recherche exploratoire du sens." (Delisle 1980:109) (Les italiques sont les siennes).

Une segmentation de l'extrait (12) du fichier Translog de Christine fondée sur des pauses minimum de 5 secondes permet d'obtenir la représentation graphique suivante:

(12) Données Translog

1. [Start][ResizeST][ $[$ :02.19.17]

2. [ $\star$ :01.01.10]Eti

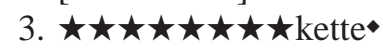

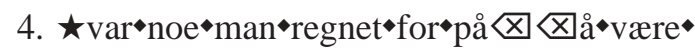

5. $\star \star$ avleggs, $\bullet$ feiet $\bullet$ bort $\bullet$ i kjølvannet $\bullet$ av $\bullet$

6. $\star \star \star 68$ • 
La dernière partie de la pause initiale de Christine, à savoir les 20 dernières secondes, consacrée à la question de savoir quel terme choisir pour traduire 'bonnes manières' dans le titre du texte, est suivie d'un triple appui sur la touche interligne, indice qu'elle s'apprête à entrer dans la phase de reformulation. Or, avant d'inscrire les trois premières lettres du mot etikette, Christine pause longuement, plus d'une minute. Il lui faudra 40 secondes supplémentaires avant qu'elle n'achève d'écrire etikette. A quoi correspondent ces deux longues premières pauses enregistrées dans la phase de reformulation?

(13) Commentaires TAP pendant la première pause

'On les croyait.' OK, il va d' abord falloir voir s'il m'est possible de garder 'on', une espèce de forme impersonnelle, ou ... Voyons voir... C'est une situation inhabituelle et je ressens un petit blocage, mais ça va sans doute s'arranger au fur et à mesure que ... (Se redresse sur sa chaise) C'est typiquement français de dire ... C'est un texte très dense. Je crois que je vais mettre etikette pour commencer et voir après comment ça fonctionne. (Prend son dictionnaire) Voyons à 'étiquette'. C'est un mot que je...

Ressentant visiblement qu'une structure syntaxique transcodée, bien que grammaticalement possible en norvégien, ne convient pas, Christine suggère d'écarter on au profit d'un sujet moins vague. Elle retient etikette, l'un des deux termes proposés initialement pour la traduction de 'bonnes manières' dans le titre. Cette solution qui introduit le référent est logique, étant donné qu'elle a décidé de reporter sa traduction du titre à plus tard.

Ses commentaires TAP pendant la deuxième pause de 40 secondes, où elle feuillète son dictionnaire, nous renseignent sur la raison pour laquelle elle a retenu le terme etikette:

(14) Commentaires TAP pendant la deuxième pause Comment s'appelle ce livre de Randi Bratholm, n'était-ce pas Etikette? Skikk og bruk? 'Le bon usage'? Non, le bon usage ça fait un peu trop... Je peux peut-être mélanger un peu, utiliser tantôt gode manerer 'bonnes manières' et tantôt... ça ira mieux quand je me serai imprégnée du texte. (Ecrit) Etikette.

Cet exemple illustre clairement que son choix des termes etikette et skikk og bruk ne découle pas d'une comparaison interlinguistique, mais d'une représentation mentale (son souvenir du livre), fruit de l'activation de son bagage cognitif pertinent.

Rendons compte d'une seconde façon de tester l'assertion de la théorie interprétative selon laquelle la comparaison interlinguistique est une méthode de traduction lacunaire, en nous appuyant sur l'exemple (15) cidessous où la juxtaposition des segments Translog 9 et 13 nous montre le rapport cognitif qu'établit Christine entre 'bonnes manières' et 'incivilités': 
(15) Données Translog

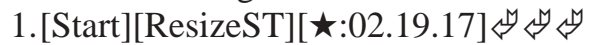

2.[ $\star: 01.01 .10] \mathrm{Eti}$

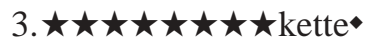

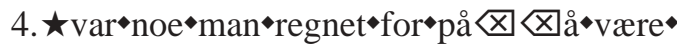

5. $\star \star$ avleggs, $\bullet$ feiet $\bullet$ bort $\bullet \bullet$ kjølvannet $\bullet$ av $\bullet$

$6 . \star \star \star 68$

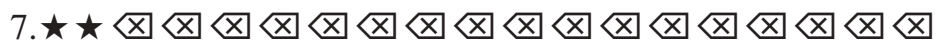

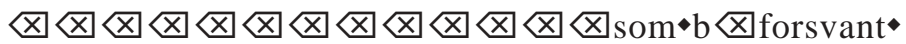
med $\bullet 9 \times \otimes 8 \bullet$ generasjonen $\bullet$

8. $\star \star \star \star \star 凶, \bullet \leftarrow \leftarrow \leftarrow \leftarrow \leftarrow \leftarrow \leftarrow \leftarrow \leftarrow \leftarrow \leftarrow \leftarrow \leftarrow \leftarrow \leftarrow \leftarrow \leftarrow \leftarrow$ $\leftarrow \leftarrow \leftarrow \leftarrow \leftarrow \leftarrow \leftarrow \leftarrow \leftarrow \leftarrow \leftarrow \leftarrow \leftarrow \leftarrow \leftarrow \leftarrow$ noe $\rightarrow \rightarrow \rightarrow \rightarrow \rightarrow \rightarrow \rightarrow$ $\rightarrow \rightarrow \rightarrow \rightarrow \rightarrow \rightarrow \rightarrow \rightarrow \rightarrow \rightarrow \rightarrow \rightarrow \rightarrow \rightarrow \rightarrow \rightarrow \rightarrow \rightarrow \rightarrow \rightarrow \rightarrow \rightarrow \rightarrow \rightarrow \rightarrow$

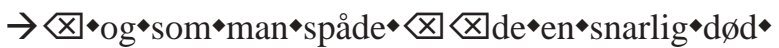

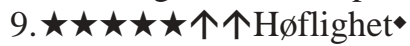

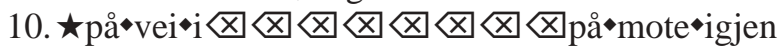

11. $\star \star \downarrow \downarrow \rightarrow \rightarrow \rightarrow \rightarrow \rightarrow \rightarrow \rightarrow \rightarrow \rightarrow \rightarrow \rightarrow \rightarrow \rightarrow \rightarrow \rightarrow \rightarrow \rightarrow \rightarrow \rightarrow \rightarrow$ $\rightarrow \rightarrow \rightarrow \rightarrow \rightarrow \rightarrow \rightarrow \rightarrow \rightarrow \rightarrow \rightarrow \rightarrow \rightarrow \rightarrow \rightarrow \rightarrow \rightarrow \rightarrow \rightarrow \rightarrow \rightarrow \rightarrow \rightarrow \rightarrow \rightarrow$ $\rightarrow \rightarrow \rightarrow \rightarrow \rightarrow \rightarrow \rightarrow \rightarrow \rightarrow \rightarrow \rightarrow \rightarrow \rightarrow \rightarrow \rightarrow \rightarrow \rightarrow \rightarrow \rightarrow$ 凶凶ख凶 $\otimes \otimes \otimes \otimes n \otimes b l e \bullet$ feid $\bullet$ bort $\bullet$ i kjølvannet $\bullet$ av $\rightarrow \rightarrow$

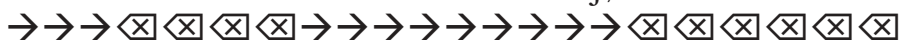
rela $\otimes\langle$ volu $\downarrow \rightarrow \rightarrow \rightarrow \rightarrow \rightarrow \rightarrow \rightarrow \rightarrow \rightarrow \rightarrow \rightarrow \rightarrow \rightarrow \rightarrow \rightarrow \rightarrow$ $\rightarrow \rightarrow \rightarrow \rightarrow \rightarrow \rightarrow \rightarrow \rightarrow \rightarrow \rightarrow \rightarrow \rightarrow \rightarrow \rightarrow \rightarrow \rightarrow \rightarrow \rightarrow \rightarrow \rightarrow \rightarrow \rightarrow$. etterhvert॰som•

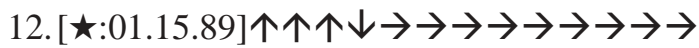

13. $\star \star \downarrow \downarrow \downarrow \downarrow \rightarrow \rightarrow \rightarrow \rightarrow \rightarrow \rightarrow \rightarrow \rightarrow \rightarrow \rightarrow \rightarrow \rightarrow \rightarrow \rightarrow \rightarrow \rightarrow \rightarrow \rightarrow$ $\rightarrow \rightarrow \rightarrow \rightarrow \rightarrow \rightarrow \rightarrow \rightarrow \rightarrow \rightarrow \rightarrow \rightarrow \rightarrow \rightarrow \rightarrow \rightarrow \rightarrow \rightarrow \rightarrow \rightarrow \rightarrow \rightarrow \rightarrow \rightarrow \rightarrow$ $\rightarrow \rightarrow \rightarrow \rightarrow \rightarrow \rightarrow \rightarrow \rightarrow \rightarrow \rightarrow \rightarrow \rightarrow \rightarrow \rightarrow \rightarrow \rightarrow \rightarrow \rightarrow \rightarrow \rightarrow \rightarrow \rightarrow \rightarrow \rightarrow \rightarrow$ $\rightarrow \rightarrow \rightarrow \rightarrow \rightarrow \rightarrow \rightarrow \rightarrow \rightarrow \rightarrow \rightarrow \rightarrow \rightarrow \rightarrow \rightarrow \rightarrow \rightarrow \rightarrow \rightarrow \rightarrow \rightarrow \rightarrow \rightarrow \rightarrow \rightarrow$ $\rightarrow \rightarrow \rightarrow \rightarrow \rightarrow \rightarrow \rightarrow \rightarrow \rightarrow \rightarrow \rightarrow \rightarrow \rightarrow \rightarrow \rightarrow \rightarrow \rightarrow \rightarrow \rightarrow \rightarrow \rightarrow \rightarrow \rightarrow \rightarrow \rightarrow$ $\rightarrow \rightarrow \rightarrow \rightarrow \rightarrow \rightarrow \rightarrow \rightarrow \rightarrow \rightarrow \rightarrow \rightarrow \rightarrow \rightarrow \rightarrow \rightarrow \rightarrow \rightarrow \rightarrow \rightarrow \rightarrow \rightarrow \rightarrow \rightarrow \rightarrow$ $\rightarrow \rightarrow \rightarrow \rightarrow \rightarrow \rightarrow \bullet$ uhøflighet

Un transcodage français faisant abstraction des corrections d'ordre orthographique et grammatical effectuées pendant l'activité d'écriture donne:

(16) Données Translog transcodées

1. Pause initiale

2. L'éti

3. quette

4. quelque chose que l'on considérait comme

5. démodé, balayé dans le sillage

6. 68

7. qui a disparu avec la génération 68

8. et auquel on promettait une mort imminente

9. La politesse 
10. de retour/en vogue à nouveau

11. balayée dans le sillage de la révolution au fur et à mesure que

12. Pause non suivie d'un segment textuel

13. l'impolitesse

Avant de terminer la traduction de la première phrase, Christine remonte au titre: voir les deux flèches $\uparrow \uparrow$ dans le segment Tanslog 9 dans (15) cidessus. La pause de 25 secondes qui précède ces deux flèches correspond aux commentaires TAP suivants:

(17) Données TAP

Politesse: ça se dit ça? La politesse en voie de disparition. La politesse a disparu à nouveau. A nouveau, mais si ça se dit. 'Retour'. A disparu. Oui, c'est bien ça. La politesse a disparu.

Elle inscrira sur son écran:

(18) Høflighet på vei i/på mote igjen

(19) La politesse de retour/en vogue à nouveau

Christine n'a donc recours ni à etikette 'étiquette', ni à gode manerer 'bonnes manières', mais à l'hyponyme høflighet 'politesse' pour traduire 'bonnes manières', terme qui en l'occurrence l'inspirera à retenir l'antonyme de politesse: uhфflighet 'impolitesse' pour traduire 'incivilités'. Ce rapport antonymique est si fort que malgré ses nombreux efforts cognitifs (documentés dans ses données TAP, dont l'analyse reste cependant à faire), en vue de trouver un terme plus approprié que uhøflighet (elle avoue que ce terme lui déplaît), c'est bien celui qu'elle adopte.

\section{Conclusion}

L'étude du processus traductionnel à partir de l'analyse de données in vivo semble avantageuse aussi bien pour la traductologie que pour la linguistique cognitive. Elle fournit à la traductologie des données empiriques précieuses sur les effets que la compréhension des idées a sur le décuplement des moyens linguistiques mis à la disposition du traducteur au niveau de la réexpression du sens du message original. Elle offre aussi des indices de l'activité cognitive des sujets traduisants susceptibles de tester de nombreuses hypothèses sur une base empirique. D'autre part elle offre un poste d'observation privilégié à la linguistique cognitive. En effet, la diversité des formulations retenues dans la langue d'arrivée pour traduire un énoncé permet de donner une idée de la multiplicité des configurations cognitives évoquées par l'expression métaphorique dans la langue de départ. De son côté, le modèle de la métaphore conceptuelle semble prometteur, car il fournit à la traductologie les instruments qui manquaient jusqu'à présent pour tenter de pénétrer la boîte noire du traducteur et d'expliquer comment est saisie et représentée une expression métaphorique. 


\section{Bibliographie:}

Albert, Laurence (2000). "Société." L'Express, janvier 4-11.

Bernadini, Silvia (2000). "Think-aloud protocols in translation research: Achievements, Limits, Future Prospects." Paper presented at UMIST/UCL Conference on Research Models in Translation Studies (University of Manchester 28-30 April 2000).

Delisle, Jean (1984). L'analyse du discours comme méthode de traduction. Ottawa: Presses de l'Université d'Ottawa.

Gibbs, Raymond W. (1994). The Poetics of Mind. Cambridge: Cambridge University Press.

Jakobsen, Arnt Lykke \& Lasse Schou (1999). “Translog documentation.” G. Hansen (éd.) (1999). Probing the process in translation: methods and results. Copenhagen: Copenhagen Business School, 73-101.

Jakobsen., Arnt Lykke (1999). "Logging time delay in translation." G. Hansen (éd.) (1999). Probing the process in translation: methods and results. Copenhagen: Copenhagen Business School, 73-101.

Jakobsen, Arnt Lykke (2000). "Understanding the Process of Translation: The Contribution of Time-Delay Studies.” B. Englund Dimitrova (éd.) (2000). Översättning och tolkning. Stockholm: ASLA:s skriftserie 12, 155-172.

Jakobsen, Arnt Lykke (2003 à venir). "Translation drafting by professional translators and by translation students." A paraître dans Traducción \& Communicación.

Jensen, Astrid (2000). The Effects of Time on Cognitive Processes and Strategies in Translation. PhD thesis. Copenhagen Business School.

Jääskelaïnen, Riitta (1987). What happens in a Translation Process: Think-aloud Protocols of Translation. Joensuu: University of Joensuu, Savonlinna School of Translation Studies.

Jääskelaïnen, Riitta (2002). "Think-aloud Protocol Studies into translation: an annotated bibliography." A paraître dans Target 14 (1), 107-136.

Lakoff, George \& Mark Johnsen (1984). Metaphors We Live By. Chicago/London: The University of Chicago Press.

Lakoff, George \& Mark Turner (1989). More than cool reason. A field to poetic metaphor. Chicago/London: The University of Chicago Press.

Langacker, Ronald (1991). Concept, Image, and Symbol - The Cognitive Basis of Grammar. Berlin/New York: Mouton de Gruyter.

Lederer, Marianne (1994). La traduction aujourd'hui - Le modèle interprétatif. Paris: Hachette.

Rydning, Antin Fougner (2001). "The synecdochial device applied to translation seen against the conceptual metaphor theory (CMT).” H. Dørum, K. Nilsson \& S. Schult Ulriksen. (éd.) (2001). Romansk Forum 14, 61-73.

Rydning, Antin Fougner (2002). "Concept métaphorique et expression métaphorique dans une perspective cognitiviste." H. Dørum (éd.) (2002). Romansk Forum 16, 723-733. http://www.digbib.uio.no/roman/page21.html

Rydning, Antin Fougner (2003 à venir). "Analyse in vivo des processus mentaux du traducteur." A paraître dans La théorie interprétative de la traduction. Regards croisés.

Séguinot, Candace (1989). The Translation Process. Toronto: H.G. Publications.

Tirkkonen-Condit, Sonja (1989). "Professional vs. Non-professional Translation: A Think-aloud Protocol Study." C. Séguinot (éd.) (1989). The Translation Process. Toronto: H.G. Publications, 73-85. 
${ }^{1}$ Les données Translog bien qu'utilisées qualitativement ici, se prêtent aussi à l'analyse quantitative pour rendre compte p. ex. de la rapidité de traduction des sujets traduisants, de leur rythme cognitif, de la longueur des segments traités, des rapports entre les trois phases de la traduction: phase d'orientation intiale, phase d'écriture, phase de révision. 